\title{
Expression of WNT-5a and ROR2 correlates with disease severity in osteosarcoma
}

\author{
BEI-JI LU ${ }^{1,2}$, YUN-QING WANG ${ }^{2}$, XUE-JIE WEI ${ }^{2}$, LIANG-QUN RONG ${ }^{2}$, DONG WEI $^{2}$, \\ CHANG-MING YAN ${ }^{2}$, DENG-JIE WANG ${ }^{2}$ and JUN-YING SUN ${ }^{1}$ \\ ${ }^{1}$ Department of Orthopedics, The First Affiliated Hospital, Soochow University, Suzhou, \\ Jiangsu 215006; ${ }^{2}$ Department of Orthopedics, the Second Affiliated Hospital, \\ Xuzhou Medical College, Xuzhou, Jiangsu 221000, P.R. China
}

Received November 7, 2011; Accepted January 16, 2012

DOI: $10.3892 / \mathrm{mmr} .2012 .772$

\begin{abstract}
Osteosarcoma, a common malignancy primarily affecting children, generally has a poor prognosis. Novel diagnostic, prognostic and therapeutic markers are required to ameliorate the negative outcomes of this disease. We investigated two potential markers, WNT-5a and ROR2, which are hypothesized to dysregulate WNT signaling pathways to promote tumorigenesis in other types of cancer. We investigated WNT-5a and ROR2 expression using immunohistochemistry in 42 osteosarcoma and 12 osteochondroma specimens, and compared the expression of these proteins with one another as well as with clinicopathological parameters. WNT-5a was detected in 34/42 (81.0\%) cases and ROR2 was detected in $31 / 42(73.8 \%)$ cases, significantly higher than in osteochondroma (16.7 and 25.0\%, respectively; both $\mathrm{P}<0.05)$. Expression of these proteins was positively correlated $(\mathrm{r}=0.552, \mathrm{P}<0.05)$. Furthermore, expression of WNT-5a and ROR2 was both correlated with Enneking surgical stage and tumor metastasis $(\mathrm{P}<0.05)$, but not with patient gender, age or pathological type. Thus, WNT-5a and ROR2 were more highly expressed in more severe disease states, and therefore may play a coordinated role in the occurrence and progression of osteosarcoma.
\end{abstract}

\section{Introduction}

Osteosarcoma is one of the most common primary bone malignancies, with an incidence of $0.3 / 100,000$ (1). The disease affects adolescents, most often males, during their rapid bone growth stage (2). Tumors typically occur at the long bone metaphysis, with distal femur and proximal tibia and humerus as the most common sites; some lesions even occur

Correspondence to: Professor Jun-Ying Sun, Department of Orthopedics, The First Affiliated Hospital, Soochow University, 188 Shizi Street, Suzhou, Jiangsu 215006, P.R. China

E-mail: sunjy0901@126.com

Key words: osteosarcoma, Wnt-5a, Ror2, immunohistochemistry, clinicopathological parameter around the knee. Unfortunately, prognosis in osteosarcoma is generally poor due to high degrees of malignancy, relapse and metastasis; the post-amputation 3 to 5-year survival rate is $<30 \%$ (3). Recent treatment advances combining neoadjuvant chemotherapy with surgery and other clinical applications have allowed the 5-year survival rates to reach 50-70\%; however, mortality and disability rates remain high (4). Continued research into potential diagnostic, prognostic and therapeutic targets may help to reduce the disability and mortality associated with this disease.

One family of genes, the WNT gene cluster, has been under investigation for its roles in cancer biology and as potential markers or therapeutic targets (5). These genes belong to a highly conserved signaling family found in a number of species from invertebrates to mammals, including humans (6). WNT proteins play a role in normal embryonic pattern formation and cell line differentiation, as well as tumorigenesis (7).

One of the most important members of this family, WNT-5a, has been linked to development of a variety of malignant tumors (8). However, this protein appears to exert different biological effects in various tumor types (9). In certain cases, WNT-5 $a$ acts as a tumor suppressor, reducing or delaying initiation, invasion and metastasis of colon cancer (10) and other malignancies (11). By contrast, the majority of studies suggest that WNT-5a acts as an oncogene, which is highly expressed in gastric cancer (12) and other malignant tumors (13). This overexpression is not only significantly related to tumor cell invasion and metastasis, but is also associated with poor prognosis and shorter survival. Furthermore, reports show that a receptor tyrosine kinase, ROR2, is capable of binding to WNT-5a to activate non-classical WNT-5a/JNK signaling pathways through non-tyrosine kinase activity. In turn, this altered signaling promotes tumor invasion and metastasis (14). WNT-5a has not been directly studied in osteosarcomas, but Morioka et al (15), in an expression profile of these tumors, found that ROR2 is upregulated in the majority of cases. Thus, WNT-5a and ROR2 represent potentially important players in the formation of these aggressive and deadly tumors and warrant further investigation.

To determine the roles of WNT-5a and ROR2 in osteosarcoma, we used immunohistochemistry to detect expression in 42 osteosarcoma samples and 12 chondroma (benign cartilage 
tumor) samples. We particularly investigated the correlation between WNT-5a and ROR2 expression, as well as the correlation between their expression and clinicopathological parameters of the tumors. These studies provide a theoretical basis for the development of, and targeted therapy for, osteosarcoma.

\section{Materials and methods}

Sample collection. Osteosarcoma specimens were collected from 42 patients undergoing surgical resection in the Second Affiliated Hospital of Xuzhou Medical College. All cases had detailed clinical data and none received preoperative chemotherapy or radiotherapy. The study population included 34 males and 8 females ranging in age from 14 to 36 years (mean age, 21.6 \pm 6.0 ). Cases were classified according to pathology as follows: fibroblast (10 cases), chondroblast (11 cases), osteoplast (16 cases) or other (mixed, giant cell or intramedullary high differentiation type; 5 cases). Additionally, cases were classified according to Enneking surgical stage (16) as follows: stage I (7 cases), stage II (27 cases), or stage III (8 cases); and according to whether they had no lymph node metastasis (17 cases) or metastasis (18 cases). In addition, 12 chondroma specimens were used as controls.

Immunohistochemistry. Tissues were fixed in neutral formalin, dehydrated and embedded in paraffin by conventional methods. Samples were sectioned $(4 \mu \mathrm{m})$ and collected on glass slides. Sections were then dewaxed with dimethylbenzene, rehydrated through an alcohol gradient, soaked, and heated for antigen retrieval. Upon cooling, 3\% hydrogen peroxide solution was used to block endogenous peroxidase activity. Slides were sealed with non-specific serum, then placed in a wet box and incubated at room temperature. Primary antibodies against WNT-5a or ROR2 [rabbit anti-human polyclonal (WNT-5a) or monoclonal (ROR2) antibodies, Santa Cruz Biotechnology] were added to the wet box prior to overnight incubation at $4^{\circ} \mathrm{C}$. Slides were washed with phosphate-buffered saline (PBS) three times prior to the addition of biotinylated secondary antibodies and incubation at room temperature. Finally, slides were washed with PBS three times prior to the addition of streptococcus avidin-peroxidase (SP kit, Beijing ZhongshanGolden Bridge Biotechnology Co., Ltd) and incubation at $37^{\circ} \mathrm{C}$ for $30 \mathrm{~min}$. DAB (Zhongshan-Golden Bridge Biotechnology Co., Ltd) was used to develop staining. Sections were counterstained with hematoxylin, dehydrated through an ethanol gradient and sealed with neutral gum. Known positive tissues were used as a positive control, and PBS was used in place of primary antibodies as a negative control.

Staining of WNT-5a and ROR2 was detected mainly in the cytoplasm. Ten high-power fields per sample were selected for analysis. The staining degree was assigned as follows: 0 for no staining detected, 1 for pale yellow staining, 2 for yellow and 3 for brown-yellow. Additionally, the percentage of positively stained cells out of the total number of tumor cells was determined and categorized as follows: $\leq 5 \%$ positive cells was scored as $0,6-25 \%$ positive cells was scored as $1,26-50 \%$ positive cells was scored as $2,51-75 \%$ positive cells was scored as 3 , and $\geq 76 \%$ positive cells was scored as 4 . The total score for each case reflects the sum of scores from
Table I. Expression of WNT-5a in osteosarcoma and osteochondroma $[\mathrm{n}(\%)]$.

\begin{tabular}{lrrrrr}
\hline Groups & $\mathrm{n}$ & - & + & ++ & +++ \\
\hline Osteosarcoma & 42 & $8(19.0)$ & $10(23.8)$ & $11(26.2)$ & $13(31.0)$ \\
Chondroma & 12 & $10(83.3)$ & $2(16.7)$ & 0 & 0 \\
Total & 54 & $18(33.3)$ & $12(22.2)$ & $11(20.4)$ & $13(24.1)$ \\
\hline
\end{tabular}

$\chi^{2}=18.643, \mathrm{p}=0.001$.

Table II. Expression of ROR2 in osteosarcoma and osteochondroma [n (\%)].

\begin{tabular}{lrrrrr}
\hline Groups & $\mathrm{n}$ & - & + & ++ & +++ \\
\hline Osteosarcoma & 42 & $11(26.2)$ & $8(19.0)$ & $12(28.6)$ & $11(26.2)$ \\
Chondroma & 12 & $9(75.0)$ & $3(25.0)$ & 0 & 0 \\
Total & 54 & $20(37.0)$ & $11(20.4)$ & $12(22.2)$ & $11(20.4)$
\end{tabular}

$\chi^{2}=12.737, \mathrm{p}=0.005$.

Table III. Correlation between WNT-5a and ROR2 expression in osteosarcoma.

\begin{tabular}{lrrrrr}
\hline & \multicolumn{5}{c}{ ROR2 } \\
\cline { 2 - 6 } WNT-5a & - & + & ++ & +++ & Total \\
\hline- & 4 & 2 & 2 & 0 & 8 \\
+ & 3 & 3 & 3 & 1 & 10 \\
++ & 4 & 2 & 2 & 3 & 11 \\
+++ & 0 & 1 & 5 & 7 & 13 \\
Total & 11 & 8 & 12 & 11 & 42 \\
\hline
\end{tabular}

$\mathrm{r}=0.546, \mathrm{p}<0.01$.

the staining degree and the proportion of positively stained cancer cells, with total scores of 0 noted as (-), 1-2 as (+), 3-5 as (++) and 6-7 as (+++).

Statistical methods. SPSS 17.0 statistical software was used for statistical analysis. The $\chi^{2}$ test was used to compare WNT-5a and ROR2 expression between groups, and Spearman rank correlation was used to analyze the relationship between WNT-5a and ROR2 expression. Analyses were two-sided, with an $\alpha$ level of 0.05 and $\mathrm{p}<0.05$ considered to indicate a statistically significant difference.

\section{Results}

WNT-5a and ROR2 are highly expressed in osteosarcoma. WNT-5a was positively expressed in $81.0 \%$ of osteosarcoma samples, compared with just $16.7 \%$ of chondromas (Table I); this difference was statistically significant $\left(\chi^{2}=18.643\right.$; 
Table IV. Correlation between expression of WNT-5a protein and clinicopathological parameters in osteosarcoma [n (\%)].

\begin{tabular}{|c|c|c|c|c|c|c|c|}
\hline Clinicopathological parameters & $\mathrm{n}$ & - & + & ++ & +++ & $\chi^{2}$ & $\mathrm{P}$ \\
\hline \multicolumn{8}{|l|}{ Gender } \\
\hline Male & 34 & $6(17.6)$ & 7 (20.6) & $10(29.4)$ & $11(32.4)$ & \multirow[t]{2}{*}{1.782} & \multirow[t]{2}{*}{0.619} \\
\hline Female & 8 & $2(25.0)$ & $3(37.5)$ & $1(12.5)$ & $2(25.0)$ & & \\
\hline \multicolumn{8}{|l|}{ Age } \\
\hline$\leq 20$ years & 25 & $2(8.0)$ & $7(28.0)$ & $6(24.0)$ & $10(40.0)$ & \multirow[t]{2}{*}{6.160} & \multirow[t]{2}{*}{0.104} \\
\hline$>20$ years & 17 & $6(35.3)$ & $3(17.6)$ & $5(29.4)$ & $3(17.6)$ & & \\
\hline \multicolumn{8}{|l|}{ Pathological type } \\
\hline Fibroblast & 10 & $1(10.0)$ & $5(50.0)$ & $4(40.0)$ & 0 & \multirow[t]{4}{*}{12.605} & \multirow[t]{4}{*}{0.181} \\
\hline Chondroblast & 11 & $3(27.3)$ & $2(18.2)$ & $1(9.1)$ & $5(45.5)$ & & \\
\hline Osteoplast & 16 & $3(18.8)$ & $3(18.8)$ & $5(31.3)$ & $5(31.3)$ & & \\
\hline Other & 5 & $1(20.0)$ & 0 & $1(20.0)$ & $3(60.0)$ & & \\
\hline \multicolumn{8}{|l|}{ Enneking surgical stage } \\
\hline I & 7 & $5(71.4)$ & $1(14.3)$ & $1(14.3)$ & 0 & \multirow[t]{3}{*}{23.607} & \multirow[t]{3}{*}{0.001} \\
\hline II & 27 & $3(11.1)$ & $9(33.3)$ & $8(29.6)$ & $7(25.9)$ & & \\
\hline III & 8 & 0 & 0 & $2(25.0)$ & $6(75.0)$ & & \\
\hline \multicolumn{8}{|l|}{ Tumor metastasis } \\
\hline Yes & 34 & $8(23.5)$ & $10(29.4)$ & $9(26.5)$ & $7(20.6)$ & \multirow[t]{2}{*}{10.435} & \multirow[t]{2}{*}{0.015} \\
\hline No & 8 & 0 & 0 & $1(25.0)$ & $6(75.0)$ & & \\
\hline
\end{tabular}

Table V. Correlation between expression of ROR2 and clinicopathological parameters in osteosarcoma [n (\%)].

\begin{tabular}{|c|c|c|c|c|c|c|c|}
\hline Clinicopathological parameters & $\mathrm{n}$ & - & + & ++ & +++ & $\chi^{2}$ & $\mathrm{P}$ \\
\hline \multicolumn{8}{|l|}{ Gender } \\
\hline Male & 34 & $9(26.5)$ & 7 (20.6) & $8(23.5)$ & $10(29.4)$ & 2.523 & 0.471 \\
\hline Female & 8 & $2(25.0)$ & $1(12.5)$ & $4(50.0)$ & $1(12.5)$ & & \\
\hline \multicolumn{8}{|l|}{ Age } \\
\hline$\leq 20$ years & 25 & $5(20.0)$ & $5(20.0)$ & $7(28.0)$ & $8(32.0)$ & 1.736 & 0.629 \\
\hline$>20$ years & 17 & $6(35.3)$ & $3(17.6)$ & $5(29.4)$ & $3(17.6)$ & & \\
\hline \multicolumn{8}{|l|}{ Pathological type } \\
\hline Fibroblast & 10 & $3(30.0)$ & $3(30.0)$ & $3(30.0)$ & $1(10.0)$ & 4.421 & 0.882 \\
\hline Chondroblast & 11 & $2(18.2)$ & $2(18.2)$ & $4(36.4)$ & $3(27.3)$ & & \\
\hline Osteoplast & 16 & $5(31.3)$ & $2(12.5)$ & $3(18.8)$ & $6(37.5)$ & & \\
\hline Other & 5 & $1(20.0)$ & $1(20.0)$ & $2(40.0)$ & $1(20.0)$ & & \\
\hline \multicolumn{8}{|l|}{ Enneking surgical stage } \\
\hline I & 7 & $5(71.4)$ & $1(14.3)$ & $1(14.3)$ & 0 & 20.472 & 0.002 \\
\hline II & 27 & $6(22.2)$ & 7 (25.9) & $9(33.3)$ & $5(18.5)$ & & \\
\hline III & 8 & 0 & 0 & $2(25.0)$ & $6(75.0)$ & & \\
\hline \multicolumn{8}{|l|}{ Tumor metastasis } \\
\hline Yes & 34 & $11(32.4)$ & $8(23.5)$ & $10(29.4)$ & $5(14.7)$ & 13.504 & 0.004 \\
\hline No & 8 & 0 & 0 & $2(25.0)$ & $6(75.0)$ & & \\
\hline
\end{tabular}

$\mathrm{P}<0.05)$. Similarly, ROR2 was positively expressed in $73.8 \%$ of osteosarcoma samples and only $25.0 \%$ of chondroma samples (Table II); this difference was also statistically significant $\left(\chi^{2}=12.737 ; \mathrm{P}<0.05\right)$. Additionally, WNT-5a and ROR2 expression in osteosarcoma were positively correlated with one another $(\mathrm{r}=0.546, \mathrm{P}<0.05$; Table III).
WNT-5a and ROR2 expression correlate with clinicopathological parameters. We investigated whether the changes in expression of WNT-5a and ROR2 may be related to the severity of osteosarcoma by analyzing various clinical and pathological parameters of the tumors. Expression of WNT-5a and ROR2 correlated with Enneking surgical stage and tumor 
metastasis (both $\mathrm{P}<0.05$; Tables IV and $\mathrm{V}$ ), but not with gender, age or pathological type.

\section{Discussion}

Changes in the WNT signaling pathway have been associated with tumorigenesis (8). Given previous findings of upregulated Ror2 (15), the product of which binds to WNT-5a (14) in osteosarcoma, we hypothesized that upregulation of this pathway promotes tumor formation and progression. Using immunohistochemistry to detect WNT-5a and ROR2 in osteosarcoma samples, we found that both proteins are more commonly expressed in the tumors, suggesting a dysregulation of the signaling pathway. Furthermore, the expression of these proteins was correlated to one another within the samples, supporting the data that indicate that ROR2 acts as a coreceptor for WNT-5a. Most notably, the expression of these proteins correlated with Enneking surgical stage and tumor metastasis, with higher expression corresponding to greater disease severity. These findings indicate that the WNT-5a signaling pathway was upregulated during osteosarcoma genesis. WNT-5a, then, acts as an oncogene in the development and progression of osteosarcoma, similar to its role in other malignancies $(17,18)$.

ROR2, as a receptor tyrosine kinase, is very important in regulating the cell cycle, migration, proliferation and differentiation (19). Misfolding of ROR2 is associated with a number of human diseases, for example, dominant brachydactylia and recessive Robinow (20). Mikels and Nusse (21) speculated that ROR2/WNT-5a binding may trigger ROR2-mediated nonclassical WNT-5a/JNK signaling pathways to promote tumor invasion and metastasis. However, few studies have demonstrated any correlation between ROR2 and tumorigenesis, particularly osteosarcoma. Thus, our results support the hypothesis that ROR2 binds WNT-5a to promote tumor formation and metastasis, likely through non-classical signaling pathways.

In conclusion, WNT-5a and ROR2 expression are significantly increased in osteosarcoma and promote formation and progression of these tumors. Thus, WNT-5a and ROR2 represent novel reference markers for evaluating osteosarcomas, and may serve as important tools in determining prognosis and treating patients with these tumors.

\section{References}

1. Unni KK: Osteosarcoma of bone. J Orthop Sci 3: 287-294, 1998.

2. Lewis VO: What's new in musculoskeletal oncology. J Bone Joint Surg Am 91: 1546-1556, 2009.
3. Wafa $\mathrm{H}$ and Grimer RJ: Surgical options and outcomes in bone sarcoma. Expert Rev Anticancer Ther 6: 239-248, 2006.

4. Meyers PA, Schwartz CL, Krailo MD, et al: Osteosarcoma: the addition of muramyl tripeptide to chemotherapy improves overall survival - a report from the Children's Oncology Group. J Clin Oncol 26: 633-638, 2008.

5. Yao H, Ashihara E and Maekawa T: Targeting the Wnt/ $\beta$ catenin signaling pathway in human cancers. Expert Opin Ther Targets 15: 873-887, 2011

6. Sylvie J, Ellen C and Kris V: The role of Wnt in cell signaling and cell adhesion during early vertebrate development. Front Biosci 17: 2352-2366, 2011

7. Clark CC, Cohen I, Eichstetter I, Cannizzaro LA, McPherson JD, Wasmuth JJ and Iozzo RV: Molecular cloning of the human proto-oncogene Wnt-5A and mapping of the gene (WNT5A) to chromosome 3p14-p21. Genomics 18: 249-260, 1993.

8. Pukrop T and Binder C: The complex pathways of Wnt $5 \mathrm{a}$ in cancer progression. J Mol Med (Berl) 86: 259-266, 2008.

9. McDonald SL and Silver A: The opposing roles of Wnt-5a in cancer. Br J Cancer 101: 209-214, 2009.

10. Dejmek J, Dejmek A, Säfholm A,Sjölander A and Andersson T: Wnt-5a protein expression in primary Dukes B colon cancers identifies a subgroup of patients with good prognosis. Cancer Res 65: 9142-9146, 2005.

11. Kremenevskaja N, von Wasielewski R, Rao AS, Schöfl C, Andersson T and Brabant G: Wnt-5a has tumor suppressor activity in thyroid carcinoma. Oncogene 24: 2144-2154, 2005.

12. Kurayoshi M, Oue N, Yamamoto H, et al: Expression of Wnt-5a is correlated with aggressiveness of gastric cancer by stimulating cell migration and invasion. Cancer Res 66: 10439-10448, 2006.

13. Blanc E, Goldschneider D, Douc-Rasy S, Bénard J and Raguénez G: Wnt-5a gene expression in malignant human neuroblasts. Cancer Lett 228: 117-123, 2005.

14. Oishi I, Suzuki H, Onishi N, et al: The receptor tyrosine kinase Ror2 is involved in non-canonical Wnt-5a/JNK signalling pathway. Genes Cells 8: 645-654, 2003.

15. Morioka K, Tanikawa C, Ochi K, et al: Orphan receptor tyrosine kinase ROR2 as a potential therapeutic target for osteosarcoma. Cancer Sci 100: 1227-1233, 2009.

16. Enneking WF, Spanier SS and Goodman MA: A system for the surgical staging of musculoskeletal sarcoma. Clin Orthop Relat Res: 4-18, 2003.

17. Huang CL, Liu D, Nakano J, et al: Wnt-5a expression is associated with the tumor proliferation and the stromal vascular endothelial growth factor - an expression in non-small-cell lung cancer. J Clin Oncol 23: 8765-8773, 2005.

18. Liu XH, Pan MH, Lu ZF, et al: Expression of Wnt-5a and its clinicopathological significance in hepatocellular carcinoma. Dig Liver Dis 40: 560-567, 2008.

19. Schlessinger J: Cell signaling by receptor tyrosine kinase. Cell 103: 211-225, 2000.

20. Afzral AR and Jeffery S: One gene, two phenotypes: ROR2 mutations in autosomal recessive Robinow syndrome and autosomal dominant brachydactyly type B. Hum Mutat 22: 1-11, 2003.

21. Mikels AJ and Nusse R: Purified Wnt5a protein activates or inhibits beta-catenin-TCF signaling depending on receptor context. PLoS Biol 4: e115, 2006. 